\title{
A MULTISPECTRAL WHOLE-HAND BIOMETRIC AUTHENTICATION SYSTEM
}

\author{
Robert K. Rowe ${ }^{1}$,Umut Uludag ${ }^{1}$, Meltem Demirkus ${ }^{2}$, Sujan Parthasaradhi ${ }^{1}$, Anil K. Jain ${ }^{2}$ \\ ${ }^{1}$ Lumidigm Inc. \\ \{rkrowe, uuludag, sparthasaradhi\}@lumidigm.com \\ ${ }^{2}$ Michigan State University \\ \{demirkus,jain\}@cse.msu.edu
}

\begin{abstract}
We describe the design and development of a prototype whole-hand imaging system. The sensor is based on multispectral technology that is able to provide hand shape, fingerprints and palmprint modalities of a user's hand by a single user interaction with the sensor. A clear advantage of our system over other unimodal sensors for these modalities include: (i) faster acquisition time, (ii) better quality images, and (iii) ability to provide spoof detection. Initial results on a medium-size database show good recognition performance based on individual modalities as well as after fusing multiple fingers and fusing finger and palm. The prototype is being refined in order to improve performance even further.
\end{abstract}

\section{INTRODUCTION}

Multibiometric systems utilize several biometric traits (e.g., fingerprint, face, iris, etc.) together in order to increase the overall system recognition accuracy, decrease the false rejects and increase user convenience. A comprehensive overview of such systems can be found in [1]. Multibiometric systems can be differentiated based on the specific traits they fuse (e.g., fingerprint and face), on the level of fusion taking place (e.g., matching score level, decision level), and on the method of fusing the multiple sources of information together (e.g., majority voting, score averaging).

We report on the design and development of a multibiometric sensor that captures multispectral images of the user's entire hand. The system described here is the first prototype of a sensor designed to collect useable multimodal biometric information under typical operating conditions, and additionally, in instances in which some or all of the hand is not in direct optical contact with the sensor platen. The resulting multispectral data is rich in biometric information providing multiple characteristics of the hand, including:

- All four fingerprints as well as a partial thumb print

- Major characteristics of the palm, including principal lines and wrinkles

- Palm ridges and minutiae

- Hand shape
- $\quad$ Skin texture

The potential advantage of a multibiometric system based on whole-hand imaging is that only a single user interaction is needed to collect the multibiometric data (finger, palm and hand). In addition, in our design, a single sensor can collect several different biometric traits, reducing the overall size and complexity of the multibiometric system when compared to other systems that use multiple sensors, one per trait. A comparison of the advantages of collecting different biometric traits (modalities) using the proposed multispectral sensor and conventional unimodal methods is summarized in Table 1. The multibiometric sensor described in this paper has many advantages relative to the corresponding conventional unimodal biometric acquisition methods. One minor disadvantage is an increase in computational requirements due to multispectral processing of data. However, the computational requirements are still quite tractable and will not be a factor as the sensor adopts new design methodologies and next generation of microprocessors. Further, the end-to-end transaction time of the proposed sensor is less than that of conventional unimodal acquisition (comprised of multiple, disjoint insertions for each trait) as only a single insertion and processing step is necessary.

The applications for a multibiometric whole-hand sensor that we envisage range from commercial physicalaccess applications to field deployments by various government organizations in providing a highly secure multibiometric system that is easy to use, reliable, robust and compact, and suitable for adverse environments. Further, the same system can also be used to simply acquire dermatoglyphic features of the hand, which can then be matched against latent prints in various applications.

\section{SENSOR CHARACTERISTICS}

\subsection{Principles of Operation}

A drawing of the major optical elements of the multispectral whole-hand imager is shown in Fig. 1. The illumination source is a white-light light emitting diode (LED) which directly illuminates the hand resting on the platen. A portion of this light is diffusely reflected from the hand into the imaging system, which comprises an imaging lens and a color digital imaging array. 
Table 1. A comparison of advantages of acquiring multiple biometric traits using the proposed multispectral, multibiometric sensor vs. conventional unimodal sensors.

\begin{tabular}{|l|l|l|}
\hline & $\begin{array}{l}\text { Multispectral } \\
\text { Multibiometric Method }\end{array}$ & $\begin{array}{l}\text { Conventional } \\
\text { Unimodal } \\
\text { Acquisition } \\
\text { Methods }\end{array}$ \\
\hline Fingerprints & $\begin{array}{l}\text { - } \\
\text { Robust acquisition } \\
\text { under adverse } \\
\text { conditions } \\
\text { - Integrated acquisition }\end{array}$ & $\begin{array}{l}\text { Reduced } \\
\text { computational } \\
\text { requirements }\end{array}$ \\
\hline $\begin{array}{l}\text { Palmprint } \\
\text { (major features } \\
\text { and ridges) }\end{array}$ & $\begin{array}{l}\text { - Reduced need to } \\
\text { manipulate hand or } \\
\text { pre-treat the skin } \\
\text { - Integrated acquisition }\end{array}$ & $\begin{array}{l}\text { Reduced } \\
\text { computational } \\
\text { requirements }\end{array}$ \\
\hline Hand shape & $\bullet$ Integrated acquisition & $\begin{array}{l}\text { Reduced } \\
\text { computational } \\
\text { requirements }\end{array}$ \\
\hline Skin texture & $\begin{array}{l}\text { Incorporates strong } \\
\text { spoof detection }\end{array}$ & N/A \\
\hline & $\bullet$ Integrated acquisition & \\
\hline
\end{tabular}

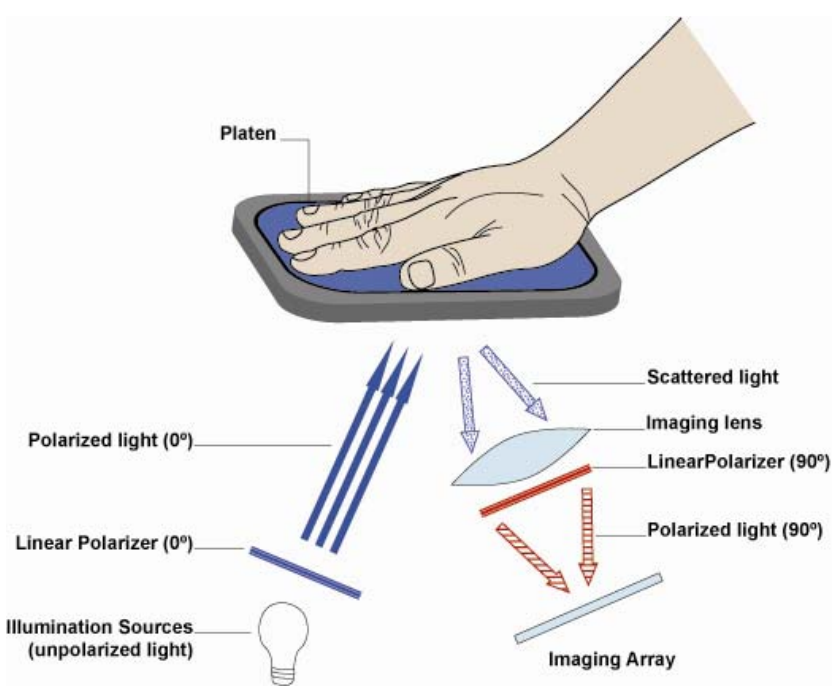

Fig. 1. Major optical components and layout of the multispectral whole-hand imaging system.

Fig. 1 also shows linear polarizers in both the illumination and imaging paths. These polarizers are oriented such that they are nearly orthogonal ("crossed") so that any light that passes through the illumination polarizer and undergoes a specular (e.g. surface) reflection into the imaging system is substantially attenuated by the imaging polarizer. This arrangement emphasizes light that has passed into the skin and been subjected to multiple optical scattering events before being imaged. In practice, as described later in this paper, the system has multiple direct illumination LEDs that turn on sequentially. Some of the LEDs do not have polarizers in front of them causing the hand to be illuminated with essentially randomly polarized light. Such an illumination state allows a greater portion of surface-reflected light to be imaged.
In addition to the polarized and unpolarized direct illumination LEDs, this system also has one additional illumination state. This illumination state uses light from LEDs that illuminate the edge of the platen. A portion of this light is trapped within the platen due to total-internalreflectance (TIR) phenomena and propagates through the platen. At points where the skin is in contact with the platen the TIR effect is negated and light is able to enter the skin. Some of the light is diffusely reflected back out of the skin into the imaging system, producing an image of the contact regions. We refer to this illumination state as TIR illumination.

A proof-of-concept system was designed and built to enable the collection of multibiometric, multispectral wholehand data. The system is illustrated in Fig. 2. The imager is a 14MP (4560x3048) CMOS color imager (CCAM, part \#FCi4-14000) coupled to a F-mount 28-75mm lens (Tamron, part \#AF09N-700). The imaging system was set to provide 500dpi resolution over a platen area of approximately 9"x6". There are 4 different, randomly polarized direct illuminators at approximately 90 degree intervals. There is also a single cross-polarized direct illuminator. All direct illuminators are 5W white-light LEDs (Lumileds, part \# LXHL-LW6C). The TIR illumination is provided by 28 T1-3/4 LEDs (Kingbright part \# WP7114RWC/J) distributed around the edge of the acrylic platen.

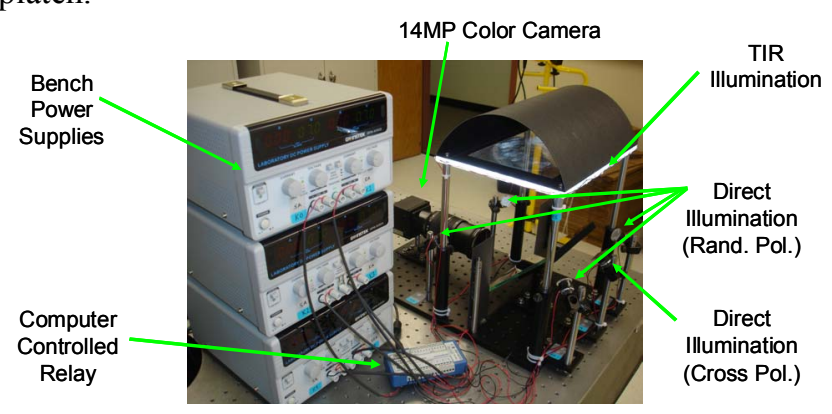

Fig. 2. Prototype multibiometric, multispectral whole-hand system.

Each time a hand is placed on the sensor platen, a series of six raw multispectral images (MSI) are collected: four images from the four unpolarized direct LEDs in four different orientations, one image collected with the crosspolarized white-light illumination, and one image collected with the TIR illumination. Each of the raw, uncompressed 8-bit images represented approximately $14 \mathrm{MB}$ of data. An example of the six raw images is shown in Fig. 3. It is important to note that conventional techniques for collecting whole-hand data are often based on just TIR methods. In those cases image data is collected only where the skin directly touches the sensor, which often represents only a small portion of the hand. The difference in coverage between direct and TIR illumination can be seen by 
comparing the TIR image (lower right) to the other five images in Fig. 3.

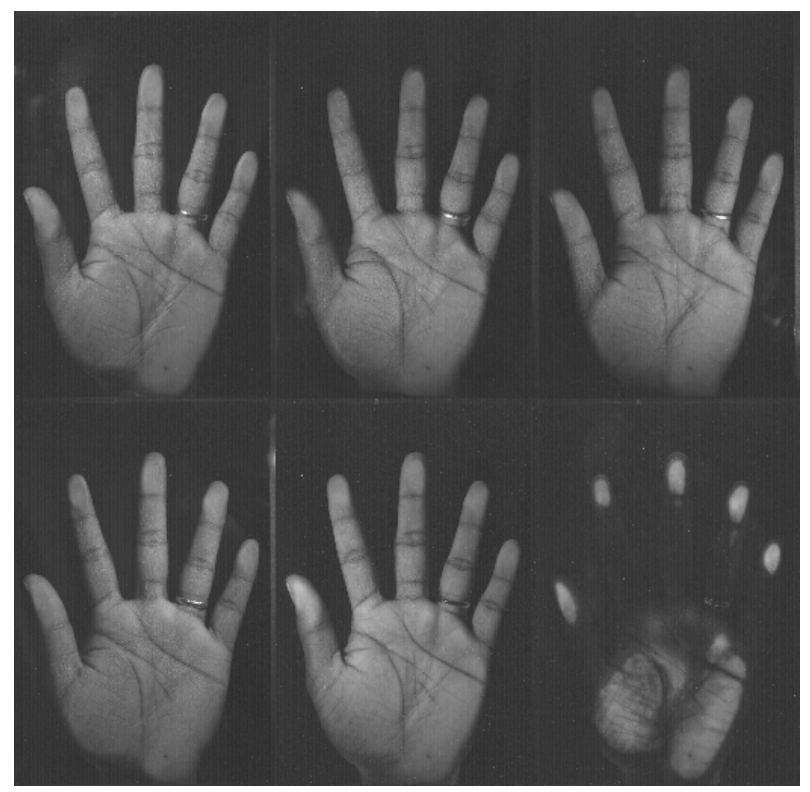

Fig. 3. Raw multispectral whole hand images. The three images in the top row as well as the lower left image correspond to unpolarized illumination conditions. The lower middle image is generated with cross polarization, and the lower right image is due to TIR illumination.

\section{MULTISPECTRAL HAND PROCESSING}

\subsection{Preprocessing}

The initial processing step for the whole-hand MSI data converts the raw Bayer data (which encodes the individual red, green and blue color plane components into a single array) into a gray-scale image using a method based on local averages of the Bayer pattern. In this approach, the original pixel values are adjusted by applying spatially varying scale factors that are derived from the ratio of the averages and a smoothed version of the original Bayer pattern. In contrast to most common methods, e.g. [2], which aim to restore the three color planes, this approach directly converts the Bayer pattern to a single gray-scale image.

The next processing step segments the image into foreground and background portions, which is needed to identify the shape and region occupied by the hand. We performed the segmentation on the five direct-illumination raw images (the TIR plane did not provide useful information for this phase) and then combined the resulting segmentations into a single, overall segmentation mask, as illustrated in Fig. 4.

Once the hand is segmented, localization of the fingertips was performed as follows: first, points on the perimeter of the binary segmentation map are found. After downsampling this list of points (in order to decrease computational time), associated curvatures are calculated, based on the cosine of angles spanned by a point and its neighbors in the list. Finally, the points with maximum curvature (that do not correspond to sections between the bases of two fingers) are identified as fingertips. The resulting $(\mathrm{x}, \mathrm{y})$ coordinates could then be used to locate a fixed-sized region $(640 \times 468)$ that localizes these fingertips, as illustrated in Fig. 5.

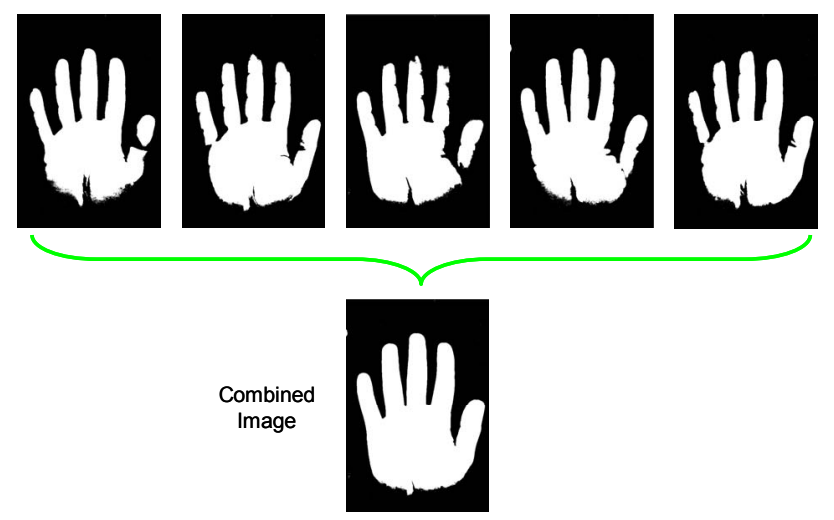

Fig. 4. Segmentation of the five individual raw images (upper row) and the resulting combined segmentation map (lower row).

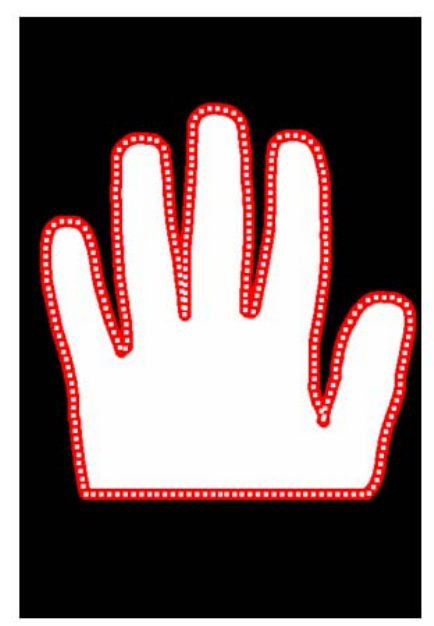

Curvature Points on Perimeter

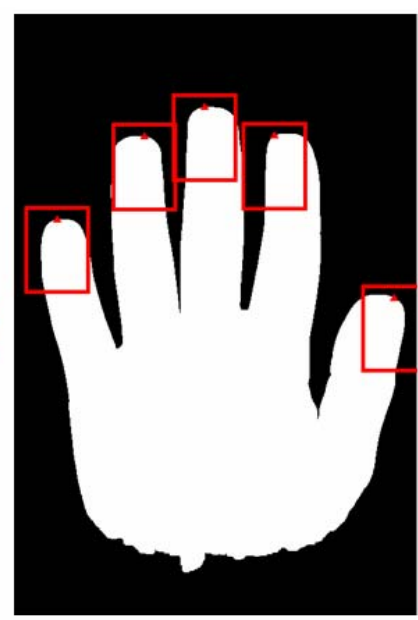

Fingerprint Locations
Fig. 5. Fingertip localization based on locations of local maxima of the curvature of perimeter map (left). The resulting localization result is shown on the right.

\section{Skin Feature Extraction}

Some of the sources of biometric signal such as fingerprints and palmprints are best represented by a single processed image generated from the MSI data rather than by the raw images directly. A method for generating such composite images was developed and applied to the wholehand data. This method is based on a modification of the wavelet fusion technique proposed in [3]. The results of generating the composite image are illustrated in Figures 6 and 7. Fig. 7, in particular, demonstrates the advantage of using multispectral techniques to extract skin features even in regions of the hand where a conventional sensing technology (TIR) fails. 


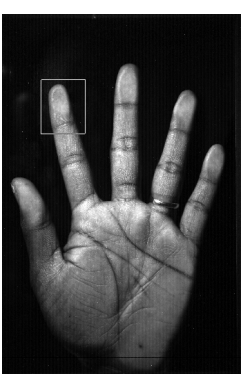

(a)

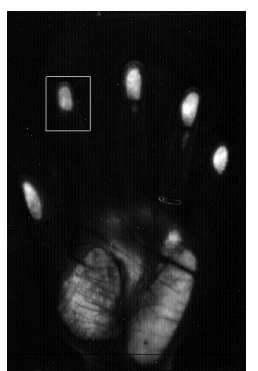

(b)

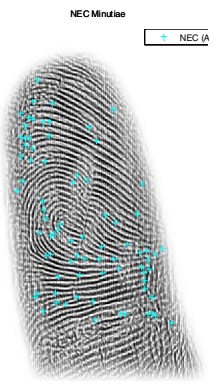

(c)
Fig. 6. Fingerprint image (c) extracted from the MSI data consisting of directly illuminated raw images (a) and a TIR image (b). The minutiae (shown in cyan) in (c) are identified by NEC fingerprint extraction software.

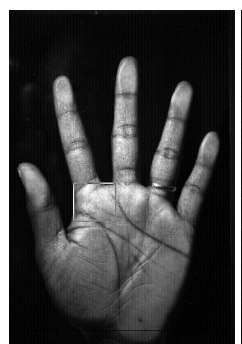

(a)

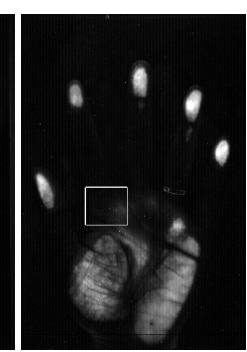

(b)

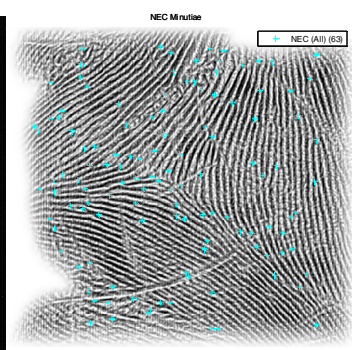

(c)
Fig. 7. Metacarpal skin feature (c) extracted from the MSI data ((a)). Note the ability of our imaging system to extract high quality features even in regions where conventional images (TIR shown in (b)) contain little or no information.

\subsection{Experimental Data}

An investigation was conducted to ensure that usable biometric information could be extracted and matched from such features. In order to demonstrate this ability, a medium-size data set was collected on 50 volunteers over two different days. During each visit that a volunteer made, 3 MSI datasets were collected for both the left and right hands. This resulted in $600 \mathrm{MSI}$ datasets corresponding to 100 unique hands.

\subsection{Fingerprint Matching}

Each of the 5 fingerprint regions on the composite images generated from the study described previously was automatically identified and the fingerprints were extracted, resulting in composite fingerprint images similar to the one illustrated in Fig. 6. Resulting fingerprint images were then processed with a commercial feature extractor (NEC PID) to identify and record a minutiae template. This algorithm is shown to be extremely accurate in third party evaluations (e.g., [4]). The generated templates were then matched to each of the other corresponding images in the dataset. Fig. 8 shows five ROC curves, one for each finger and thumb. As seen here, ring finger results in the best performance for a large section of the ROC. As expected, the performance of the thumb is degraded relative to that of the other fingers since the system captures only a partial thumbprint due to the hand placement geometry on the platen.

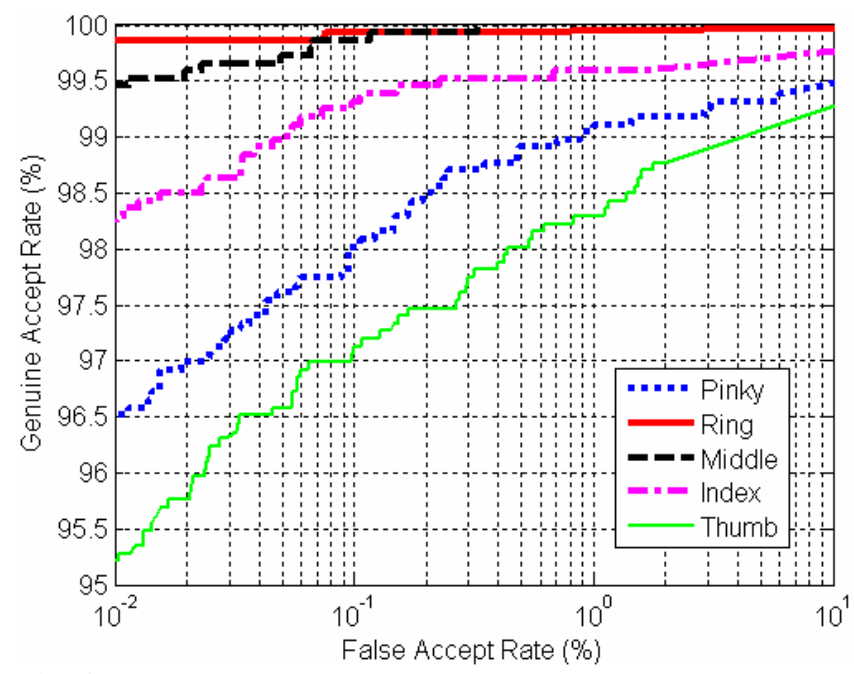

Fig. 8. ROC curves for individual fingerprint matches.

A simple method of multibiometric fusion was applied by summing the scores from all the five fingerprint matches corresponding to each pair of images. This resulted in a perfect separation of genuine and imposter matches on this dataset.

\subsection{Palmprint Matching}

Palmprints have a long history in forensics, but they have only recently been recognized as a viable and useful biometric in civilian applications. Unlike palmprint identification systems used in forensics, the systems used in access control types of applications can work effectively with low-resolution palmprint images ( 100 ppi). According to the features used for palmprint representation, we can categorize the various palmprint identification methods into three categories [5]: (i) structural feature-based, (ii) appearance-based, and (iii) texture-based. The best palmprint matching approach, in terms of authentication accuracy, was introduced by Sun et al. [5]. The main idea of this method is to compare two line-like image regions and to generate one-bit feature code representing the outcome of the comparison (at each image location). One of the advantages of using this approach is that even if the image intensities vary due to, say, non-uniform illumination, the ordinal relationship among the neighborhood regions in the image maintains some stability. We have adapted this approach in our experiments since the whole hand MSI sensor does introduce a non-uniform illumination effect across different bands of the multispectral hand image.

\section{Preprocessing}

The palmprint preprocessing stage first enhances the image contrast and binarizes the gray scale image. A single 
binary hand image is obtained by utilizing a voting scheme for each pixel in individual binarized band images. Morphological operators are then applied to remove the noise in the background and fill in the holes in the hand region, producing a binary image of the hand. This binary image helps us to remove the thumb region since it is not of interest in this phase. The mean radial distance from the points on the hand contour to the hand centroid is computed; the points with the minimum mean radial distance are selected as the anchor points.

The two anchor points that we detect help us in two major tasks: (i) alignment of hand image. The origin of the coordinate system, after the alignment, is defined to be the midpoint of two anchor (inter-finger) points: the point between index and middle finger and the point between the middle and pinky finger. The slope of the line, $\alpha$, passing through the anchor points is determined and each band image is rotated in the direction of $\alpha$ around the anchor midpoint; (ii) extracting a region of interest (ROI) that is the $1000 \times 1000$ region which lies on the horizontal line passing through the midpoint of each band image (Fig. 9); the average size of the palmprint image is $1500 \times 1300$.

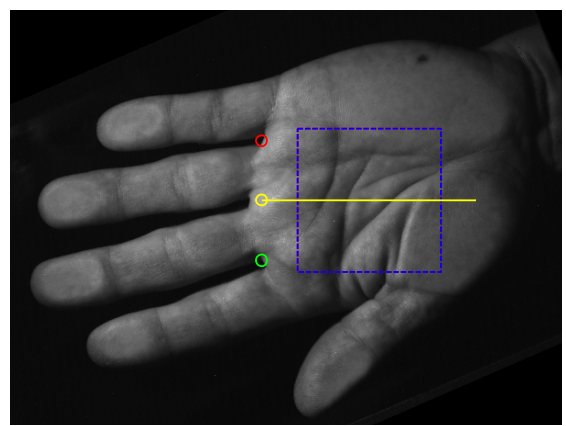

Fig. 9. The ROI in the rotated hand image, anchor points and the anchor midpoint.

Since the ordinal feature representation [5] is based on the coarse level palmprint information, we downscale the ROI image from $1000 \times 1000$ to $256 \times 256$ (Fig. 10). This reduces the feature extraction and matching time significantly.

\section{Feature Extraction}

To extract palmprint features, each ROI is filtered with an orthogonal line ordinal filter (OF) introduced in [5]

$$
O F(\theta)=f(x, y, \theta)-f(x, y, \theta+\pi / 2),
$$

where $f$ is the 2D Gaussian function with orientation $\theta$. In our experiments, we choose three filters with values of $\theta$ as $0, \pi / 6$ and $\pi / 3$; the horizontal and vertical scales of the Gaussian filter, $\delta_{\mathrm{x}}$ and $\delta_{\mathrm{y}}$, are set to 0.5 and 0.1 , respectively. The filtered images are quantized to binary images according to the sign of the filter response at each pixel (Fig. 11).

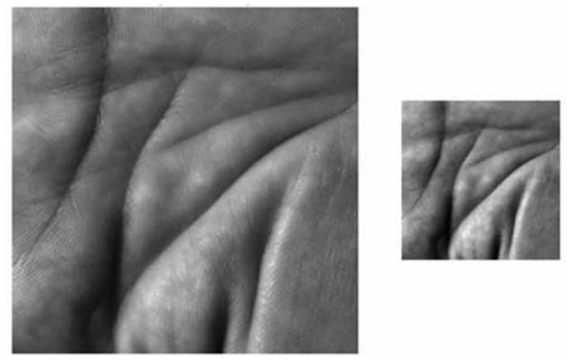

Fig. 10. The original ROI and the down-sampled ROI.

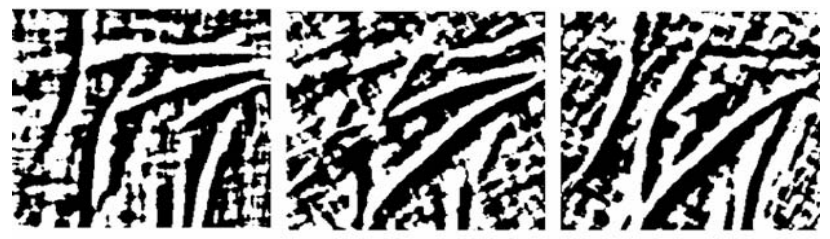

Fig. 11. Ordinal feature vectors for $\theta$ equal to $0, \pi / 6$ and $\pi / 3$ for the ROI shown in Fig. 10.

\section{Matching}

The matching of two palmprints is done separately for each raw MSI image. The dissimilarity score for each band is obtained by averaging the normalized Hamming distances of the three corresponding feature vectors. To minimize the alignment related problems, the features are translated vertically and horizontally in the range of $[-5,5]$ pixels; the minimum distance score obtained from the translated images is considered to be the final score for that specific band. Since for each palmprint pair, we have five distance scores in the range $[0,1]$, one per band, a score level fusion is needed. Among the various score level fusion methods that we tried, the product-rule provided the best performance of $91.5 \%$ genuine acceptance rate (GAR) at a false acceptance rate (FAR) of $0.01 \%$.

\subsection{Fusion of Fingerprint and Palmprint}

To demonstrate fusion of information from different biometric modalities, we chose to fuse the match scores from a single finger (ring) to the scores generated by the palms. Score level fusion of fingerprint and palmprint was achieved by employing a weighted sum-rule method (palmprint weight: 0.15, fingerprint weight: 0.85). Before summing the scores, the palm print match scores were converted from distance to similarity by multiplying them by -1 . Both fingerprint and palmprint match scores were normalized to the scale of $[0,1]$ by utilizing min-max normalization technique [1]. The resulting ROC curves corresponding to the individual finger and palm biometrics as well as the fused result are shown in Fig. 12. Fusing the information from a single fingerprint and a palm print resulted in match performance that clearly exceeds that of either of the individual biometric traits. 


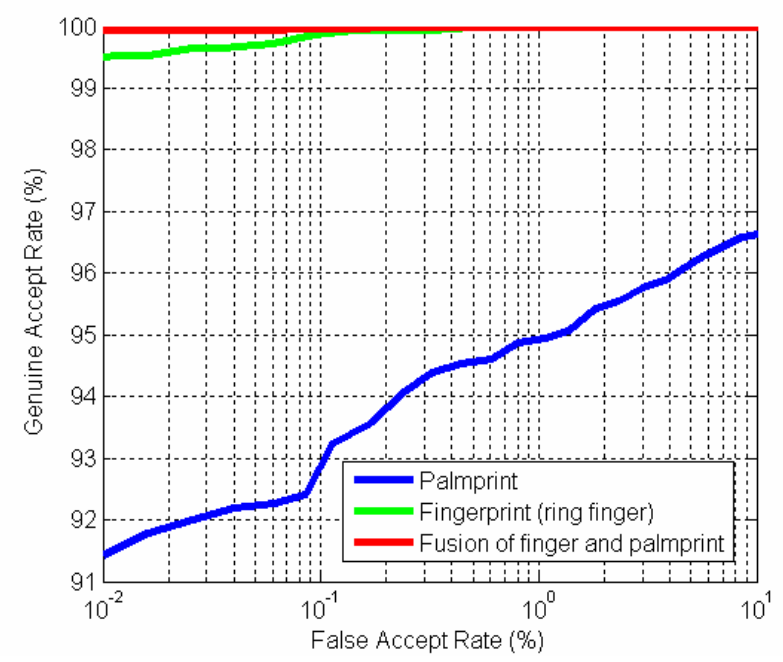

Fig. 12. ROC curve for fusion of index finger and palm.

While a number of previous attempts have been made to fuse all or a subset of palmprint, hand shape and fingerprint information [6], our device is the only one that captures all the modalities simultaneously using a single sensor/device. As an example, in [7], the palmprint and hand shape came from one sensor, but the fingerprints were captured by a different sensor. Unlike some other palmprint/hand shape sensors, our integrated sensor does not constrain the user in placement of his hand (e.g., with pegs). Another significant difference between our fusion study and previous studies involving the same three modalities is that our database is a true multimodal and not virtual one as in [7]. In other words, the same users are providing all the three biometric modalities in our database whereas in [7], the fingerprint images came from a different user population than the hand shape and palmprints. Further, the recognition results of our study are significantly better than other studies because in addition to fusion, our sensor is based on multispectral imaging technology that provides higher quality images [8].

\section{CONCLUSIONS}

A prototype of a multibiometric, multispectral whole-hand sensor system was designed, built and used to collect a medium-size set of data. Analysis of these data demonstrate that multiple biometric features can be extracted from the multispectral images of the hand including fingerprints, principle lines and wrinkles of the palm, ridges and minutia on the palm, skin texture, and hand shape. A demonstration of the ability to perform biometric fusion was conducted in two different ways. First, biometric fusion was performed across match scores produced by the five fingers, which produced perfect classification for the dataset. Second, the scores from a single finger were fused with scores generated from the major features of the palm, resulting in greatly improved performance relative to either of the individual biometrics.

Our future work will concentrate on developing and refining each of the individual biometrics as well as the fusion techniques used to combine them. Larger evaluation studies will be conducted to better estimate the performance of this multispectral multibiometric sensing system.

\section{ACKNOWLEDGEMENTS}

The authors wish to gratefully acknowledge partial support for this work received from the US Army under a Phase I SBIR, Contract \#W911NF-07-C-0022.

Lumidigm Inc.'s Sangeetha Illendula and Leesa Jenkins' contributions in the area of recruiting and collecting data from study volunteers are gratefully acknowledged.

\section{REFERENCES}

[1] A. Ross, K. Nandakumar, and A.K. Jain, Handbook of Multibiometrics, Springer Verlag, 2006.

[2] R. Ramanath, W.E. Snyder, G.L. Bilbro, and W.A. Sander, "Demosaicking methods for Bayer color arrays", Electronic Imaging, vol. 11, no. 3, pp. 306-315, 2002.

[3] P. Hill, N. Canagarajah, and D. Bull, "Image fusion using complex wavelets", Proc. $13^{\text {th }}$ British Machine Vision Conference (BMVC), pp. 487-496, 2002.

[4] C. Wilson et al., "Fingerprint vendor technology evaluation: 2003", available at: http://fpvte.nist.gov /report/

ir_7123_summary.pdf

[5] Z. Sun, T. Tan, Y. Wang, and S.Z. Li, "Ordinal palmprint representation for personal identification", Proc. IEEE Computer Vision and Pattern Recognition (CVPR), vol. 1, pp. 279-284, 2005.

[6] A. Kumar, D.C.M. Wong, H.C. Shen, and A.K. Jain, "Person verification using palmprint and hand geometry biometric" Proc. Audio- and Video-Based Biometric Person Authentication (AVBPA), pp.668-675, 2003.

[7] A. Kumar and D. Zhang, "Combining fingerprint, palmprint and hand-shape for user authentication", Proc. Int'l. Conf. Pattern Recognition (ICPR), pp. 549-552, 2006.

[8] R.K. Rowe, K.A. Nixon, and P.W. Butler, "Multispectral fingerprint image acquisition", in Advances in Biometrics: Sensors, Systems and Algorithms, N.K. Ratha and V. Govindaraju (Eds), Springer, 2007. 